\title{
Progressive anarthria with secondary Parkinsonism: a clinico-pathological case report
}

\author{
Emmanuel Broussolle, Michel Tommasi, François Mauguière, Guy Chazot
}

\begin{abstract}
The pathological process and lesion topography in patients with the syndrome of progressive aphasia are heterogeneous and few necropsy examination cases have been investigated. This is a case report of a 53 year old right handed man with progressive anarthria and secondary Parkinsonism over a period of six years. Positron emission tomography (PET) showed a decreased cerebral blood flow and metabolism in the frontal cortex, which was more pronounced on the left. Neuropathology disclosed a spongiform vacuolation in layer II of the frontal cortex, mostly in the Broca area, and neuronal loss in the substantia nigra. This original case reinforces the view that there are different entities of the syndrome of progressive aphasia which can be identified on the basis of clinical, neuroimaging and anatomical data.
\end{abstract}

Slowly progressive aphasia without dementia is a degenerative condition usually associated with cortical atrophy and hypometabolism in the left perisylvian area. ${ }^{12}$ Focal histological changes, notably a spongiform vacuolation, have been described in very few cases. ${ }^{3}$ We report a similar but distinct case combining progressive anarthria and a secondary extrapyramidal syndrome, documented by $\mathrm{CT}$, MRI, positron emission tomography (PET), and pathological findings.

\section{Case report}

In 1984 a 53 year old right handed man with a history of left nephrectomy in 1975 for urinary lithiasis, first noticed difficulties in articulating properly. In 1985 he could not continue his job as a school teacher and from 1986 became increasingly reliant on the use of written messages and sign language for communication.

From 1986-88, the patient was followed up by a speech therapist. Analysis of data from 1986, including tape recordings and written exercises, showed all elements of dysarthria and anarthria. The patient's voice was hypophonic with abnormal prosody and restricted melodic range. His speech flow was laborious, syllabic, and explosive for occlusive phonemes, with short phrases (two or three words). Phonetic disintegration included frequent sound substitutions, simplifications, additions, retrograde assimilations and antepositions of consonants, and diphthongizations of vowels. Repetition appeared increasingly impaired for syllables ( $31 / 50$ correct spelling) and simple words (73/112), to complex words (5/14). The repetition of sentences was an effort and he showed more frequent articulatory distortions with longer phrases. In the naming task, the patient was able to spell the names of 20 images out of 25 correctly, with a few paraphasic errors that occurred only for unusual words. In contrast, auditory and written comprehension tests were normal, and there were no missing words in the written exercises, or grammatical or orthographical errors.

The patient was referred to the Department of Neurology for the first time in June 1988. He could only utter a few words, but did not show any signs of aphasia. He was able to answer all oral or written questions correctly by writing. In September 1989 he was almost mute, but comprehension was normal and expression fairly preserved. Typewriting tests showed only a few literal perseverations and an occasional excess of short connecting words.

Conversely, there was no intellectual deterioration. The patient was still active in household, administrative, recreative and other activities of everyday life. The Wechsler Adult Intelligence Scale-Revised (WAIS-R) was administered in October 1988 and September 1989. Owing to the speech disability, the verbal IQ could not be quantified. The performance IQ was 89 in 1988 and 98 in 1989. The patient was easily able to find the connection between abstract words and to group them by categories. He was successful in most of the information, similitude and arithmetic tests. Visual and verbal memory were also preserved. The patient could identify the pictures of famous people, and reproduced the Rey Osterreith complex figure correctly. The 1989 scores were $33 / 36$ and $23 / 36$ for copy and recall respectively. After listening to a one page story he was able to select, from a list of $50,90 \%$ of the 25 words present in the text. The patient, however, was slow in performing the neuropsychological tests. He failed to reproduce a sequence of three hand postures or simple motor rhythms, with frequent perseverances, and to perform the Trail making test B. All these signs reflected some degree of frontal lobe dysfunction.

Another finding was the late occurrence of progressive striate rigidity, which was noted for the first time in October 1988, with a mild bradykinesia and cog-wheel rigidity in the right upper arm associated with a slight action tremor and buccofacial apraxia. Tendon and 
glabellar reflexes were brisk, with a right Babinski sign. Optic fundi, laryngoscopic examination, EEG, and electromyogram were normal. In February 1989 the patient was started on Sinemet with a dosage of $200 \mathrm{mg} /$ day orally with no clear benefit; higher doses were not tolerated. In September 1989 rigidity was present in the four limbs, though more severe in the right arm; bilateral Babinski's signs, grasp and sucking reflexes were noted with severe buccofacial apraxia but no supranuclear ophthalmoplegia.

The patient started complaining of swallowing difficulties and gait disturbances which caused several falls in December 1989. After one fall he presented, in January 1990, with a right hemiplegia related to a left subdural haematoma as diagnosed by CT. The patient died in February 1990 from pulmonary infection and renal failure after surgical evacuation of the haematoma.

\section{Results}

CT, MRI, and PET findings

Between October 1988 and September 1989, one MRI, including $\mathrm{T} 1$ and T2 weighted images, and two CT scans showed a mild
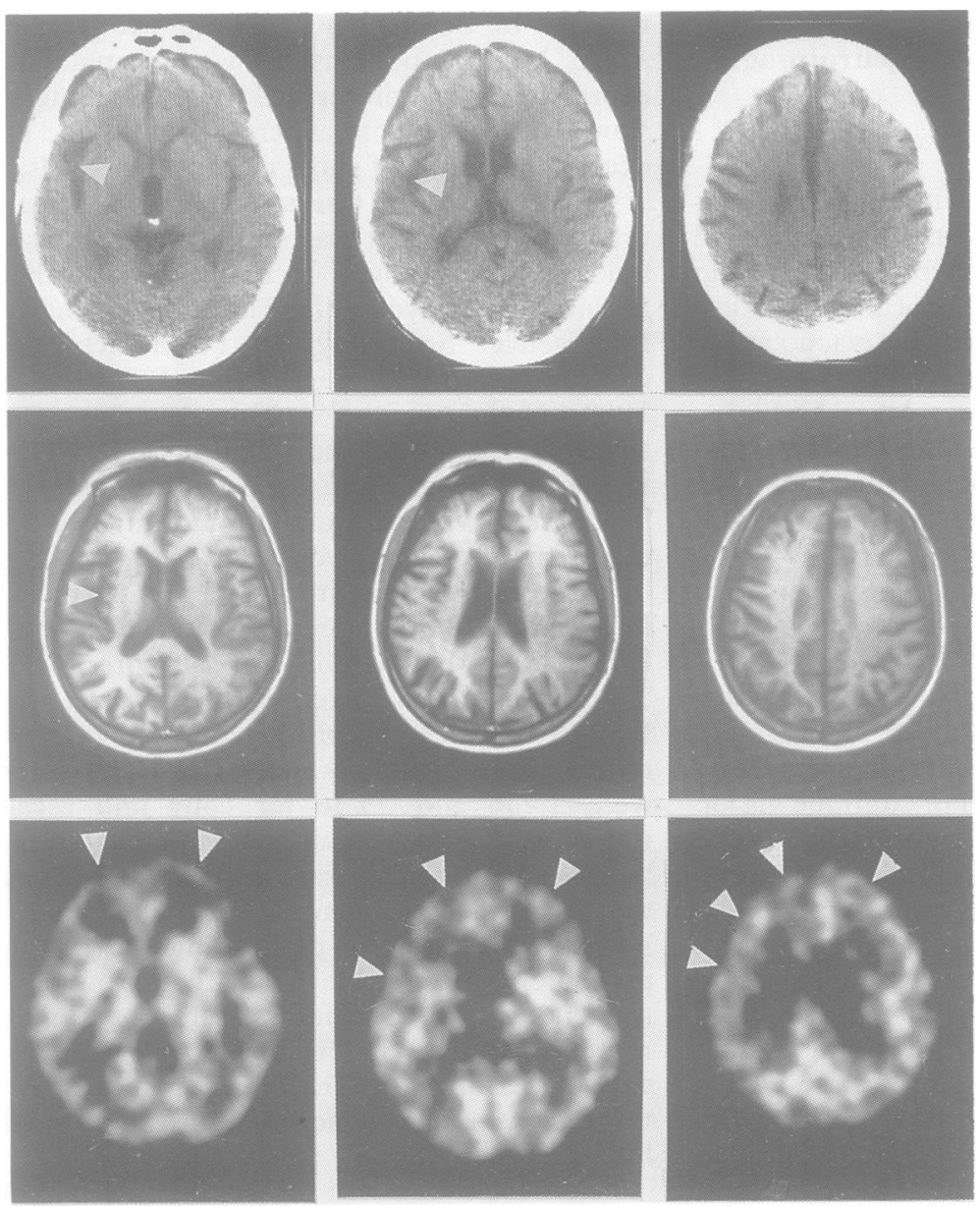

Figure $1 C T$ (upper row) and 0.5 Tesla T1-weighted MRI (middle row) images showing a mild frontal atrophy and enlargement of the left Sylvian fissure (arrow). The left hemisphere is on the left of each image. The corresponding PET images of cerebral blood flow (bottom row) were obtained $54 \mathrm{~mm}, 66 \mathrm{~mm}$ and $78 \mathrm{~mm}$ above the IOM line. $A r C B F$ decrement, more pronounced on the left than the right, was observed in most frontal regions (arrows). frontal atrophy and enlargement of the left sylvian fissure (fig 1). A PET study, using ${ }^{15} \mathrm{O} 2$ and $\mathrm{C}^{15} \mathrm{O} 2$ steady state methods, ${ }^{4}$ was performed in November 1989, with informed consent. $\mathrm{C}^{15} \mathrm{O} 2$, then ${ }^{15} \mathrm{O} 2$ were produced by a CYPRIS 325 CGR MEV cyclotron and inhaled sequentially. Once cerebral radioactivity reached a plateau, the patient was scanned using a seven slices time of flight TTV 03-LETI PET-camera (lowest plane was $18 \mathrm{~mm}$ above the inferior orbito-meatal (IOM) line; the between plane distance was $12 \mathrm{~mm}$; and axial and in plane resolution was 9 and $6.5 \mathrm{~mm}$ respectively). Regions of interest (circles of six pixels each) were drawn in cortical, subcortical, and cerebellar areas. By means of arterial blood sampling and equilibrium models, ${ }^{4}$ regional cerebral blood flow (rCBF), regional oxygen consumption (rCMRO2) and oxygen extraction fraction (OEF) were computed.

In most cortical regions outside the frontal lobes as well as in the thalamus, basal ganglia and cerebellum, the rCBF and $\mathrm{rCMRO} 2$ values were normal, ${ }^{5}$ namely in the $40-60$ and 3-4 $\mathrm{ml} / 100 \mathrm{ml} / \mathrm{min}$ ranges. The rCBF and rCMRO2, however, were reduced in the frontal lobes, with a decreased frontal occipital ratio $(0 \cdot 69)$, the lowest values being observed bilaterally in the precentral regions ( 30 and 2.5 respectively). This decrement was more pronounced on the left than the right (fig 1), particularly in the posterior part of the inferior frontal gyrus (left and right values were respectively 37.6 and 44.9 for $\mathrm{rCBF}$, and 3.2 and 3.8 for $\mathrm{rCMRO} 2$ ). There was no uncoupling between $\mathrm{rCBF}$ and $\mathrm{rCMRO} 2$, as shown by homogeneous OEF values in the $40-45 \%$ range.

\section{Pathological findings}

General pathology The heart showed atherosclerosis of the coronary arteries and an area of old scarring in the wall of the left ventricle consistent with healed posterolateral myocardial infarction. There was a pneumonia in the left lower lobe and bronchitis in the right lower lobe. The liver was hypertrophic $(2.9 \mathrm{~kg})$ but homogeneous, with mild centrilobular parenchymal atrophy and fibrosis without steatosis, secondary to chronic passive venous congestion. The right kidney showed a tubular atrophy with interstitial fibrosis secondary to pyelonephritis. There were no abnormalities in the stomach, spleen, pancreas, adrenal medulla, and pituitary gland.

Neuropathology There was slight atrophy of the frontal lobes. Coronal sections did not reveal any abnormalities in the white matter, basal ganglia and thalami. The substantia nigra was depigmented bilaterally. The cerebellum, brainstem and spinal cord were normal. Histological techniques included paraffin embedding, and haemalum-phloxine saffran, phosphotungstic acid haematoxylin (PTAH), and Bodian stainings. The most striking histological finding was a spongiform change in cortical layer II with only mild neuronal loss and astrogliosis. This was particularly pronounced in the Broca area in the left hemi- 
Figure 2 A) Superficial cortical layers of the left inferior frontal gyrus

(Broca area) depicting spongiform changes in layer II, and mild gliosis in layer I. PTAH stain. Bar: $200 \mu \mathrm{m} ; \mathrm{B})$ Schematic drawing of a coronal section of the brain at the level of the head of the caudate nuclei, showing the distribution of the spongiform changes in the cortical layer II, from predominant (arrows) (black area in the left inferior frontal gyrus including the Broca area), to moderate (dotted area in adjacent sections to the left inferior frontal gyrus and parts of the right inferior frontal gyrus) and mild changes (dotted line in most of the rest of the frontal cortex and small parts of the temporal cortex).

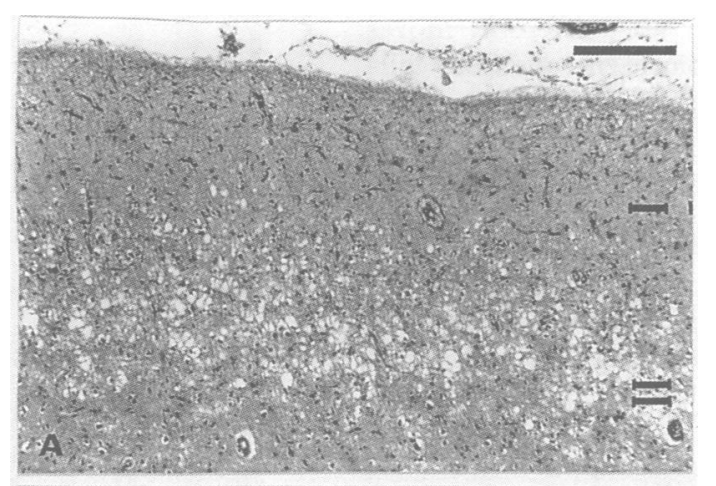

hypometabolism in the temporal cortex of the dominant hemisphere. ${ }^{129}$ Nonfluent aphasia and extrapyramidal signs, however, have been reported in a few cases. ${ }^{1{ }^{10-12}}$ In Mesulam's original report, ${ }^{1}$ patient 3 had characteristics of Broca's aphasia with buccofacial apraxia, and later became mute and akinetic. Goulding et $a l^{10}$ reported a progressive aphasia of five years duration with right-sided tremor and rigidity, and a severe atrophy and reduced blood flow in the left hemisphere. Our patient shares some clinical and metabolic similarities with this latter case. Nevertheless, none of these patients presented initially as a pure anarthria, and the underlying pathological process was unknown in all of them.

The association of focal spongiform degeneration with a neuronal loss in the substantia nigra is another remarkable feature of our case, which has not been reported in patients with progressive aphasia to date. There were no histological signs of Alzheimer's or Pick's disease in a biopsy specimen from the left superior temporal region in patient 3 of Mesulam's 1982 report. ${ }^{1}$ Focal spongiform degeneration affecting the left perisylvian region was recently reported in 2 cases of Mesulam's aphasia. ${ }^{3}$ Apart from histological changes in the substantia nigra, our case differs from these two cases in that spongiform changes were distributed more frontally with a clear fronto-occipital gradient. Association of diffuse cortical spongiosis with substantia nigra degeneration has been described by Horoupian et $a l^{13}$ in patients with dementia, amyotrophic lateral sclerosis and mild Parkinsonism, a syndrome which substantially differs from that observed in our patient. Similarly, a mild dropout of substantia nigra pigmented neurons was recently reported by Green et $a l^{14}$ in their case ${ }^{4}$ of progressive aphasia. The clinical presentation and the histological findings, however, were very different from that observed in our case, since there was global dementia and widespread cortical neuronal loss and microvacuolation.

From a series of 460 dementia patients referred to a regional brain bank, Knopman et $a l^{15}$ found 14 cases of non-Alzheimer degenerative dementia reminiscent of our case. Most patients were under the age of 70 at death, with the disease usually lasting between two and seven years. All the cases had degeneration of the cerebral cortex, characterised by vacuolation of the second cortical layer with prominent astrocytosis in the deep layers in more affected cases. This degeneration was most severe in the frontal and parietal cortex, less pronounced in the temporal cortex, and generally absent in the occipital cortex. The substantia nigra was severely degenerated in 11 cases. The clinical spectrum consisted usually of a frontal lobe dementia then rigidity, dysarthria and dysphagia. Our case undoubtedly has both clinical and pathological overlap with the syndrome of "dementia lacking disinctive histologic features", as quoted by Knopman et al. ${ }^{15}$ In this series, however, there was a positive family history for neurological disease, mainly dementia, in eight of the 14 patients. 
The initial presentation was different, moreover, with cognitive impairment, memory failure, and changes in personality, whereas our patient suffered from a pure progressive anarthria over several years and showed no clinical evidence of dementia even at an advanced stage of his illness. In addition, these authors found a degeneration of the hippocampus in most cases, and of the medical thalamus, striatum and hypoglossal nucleus in several cases, all brain regions that were unaffected in our case.

In conclusion, this case supports the view that various degenerative processes, with different topographical distributions, might account for the variability of the clinical presentation of "primary progressive aphasia" syndrome.

We thank B Schott, B Laurent, L Cinotti, D Le Bars, MM Martin, A Jouvet and B Dubois for their scientific advice; J Raymond for surgical management; and $P$ Mauger-Tête, $F$ Lavenne, MF Gravejat, JP Serrat and G Mollaret for their technical help.

1 Mesulam M-M. Slowly progressive aphasia without generalized dementia. Ann Neurol 1982;11:592-8.

2 Chawluk JB, Mesulam M-M, Hurtig $\mathrm{H}$, et al. Slowly progressive aphasia without generalized dementia: studies progressive aphasia without generalized dementia: studies
with positron emission tomography. Ann Neurol 1986; with positr.

3 Kirshner HS, Tanridag O, Thurman L, Whetsell wo. Progressive aphasia without dementia: two cases with focal spongiform degeneration. Ann Neurol 1987;22: 527-32.
4 Frackowiak RSJ, Lenzi G-L, Jones T, Heather JD. Quantitative measurement of regional cerebral blood flow and oxygen metabolism in man using $O$ and positron emission tomography: theory, procedure, and normal
values. 7 Comput Assist Tomogr 1980;4:727-36.

Leenders KL, Perani D, Lammertsma AA, et al. Cerebral blood flow, blood volume and oxygen utilization. Normal values and effect of age. Brain 1990;113:27-47.

6 Alajouanine Th, Pichot $P$, Durand M. Dissociation des Alajouanine Th, Pichot $P$, Durand M. Dissociation des
altérations phonétiques avec conservation relative de la langue la plus ancienne dans un cas d'anarthrie pure che un sujet français bilingue. Encéphale 1949;28:245-65.

7 Lecours AR, Lhermitte F. The "pure form" of the phonetic disintegration syndrome (pure anarthria): anatomoclinical report of a historical case. Brain Lang 1976, 3:88-113.

8 Gibb WRG, Luthert PJ, Marsden CD. Corticobasal degeneration. Brain 1989;112:1171-92.

9 Tyrrell PJ, Warrington EK, Frackowiak RSJ, Rossor MN. Heterogeneity in progressive aphasia due to focal cortical atrophy. A clinical and PET study. Brain 1990;113: atrophy.

10 Goulding PJ, Northen B, Snowden JS, MacDermott N, Neary D. Progressive aphasia with right-sided extrapyrNeary $D$. Progressive aphasia with right-sided extrapyramidal signs: another manifestation of localised cerebra

11 Kempler D, Metter EJ, Riege WH, Jackson CA, Benson DF, Hanson WR. Slowly progressive aphasia: three cases with language, memory, CT and PET data. $f$ Neurol Neurosurg Psychiatry 1990;53:987-93.

12 Delecluse F, Andersen AR, Waldemar G, et al. Cerebral blood flow in progressive aphasia without dementia. Case report, using 133-Xenon inhalation, Technetium 99m hexamethylpropyleneamine oxime and single photon emission computerized tomography. Brain 1990;113: 1395-404.

13 Horoupian DS, Thal L, Katzman R, et al. Dementia and motor neuron disease: morphometric, biochemical, and Golgi studies. Ann Neurol 1984;16:305-13.

14 Green J, Morris JC, Sandson J, McKeel DW Jr, Miller JW. Progressive aphasia: a precursor of global dementia? Progressive aphasia: a prec

15 Knopman DS, Mastri AR, Frey II WH, Sung JH, Rustan T. Dementia lacking distinctive histologic features: A common non-Alzheimer degenerative dementia. Neurology 1990;40:251-6. 